\title{
El trabajo del estudiante y el uso de plataforma de apoyo a la docencia, como opciones metodológicas en la universidad
}

\section{Felix Zurita Ortega ${ }^{1}$, Jose Ignacio Soto Gonzalez ${ }^{2}$, Felix Zurita Molina $^{3}$, Miguel Angel Gallardo Vigil ${ }^{4}$, Natalia Padilla Zea ${ }^{5}$}

${ }^{1}$ Departamento Didactica de la Expresión Musical, Plastica y Corporal, Facultad de Humanidades y Educacion, Universidad de Granada (Campus de Melilla).

${ }^{2}$ Escuela Universitaria de Magisterio La Inmaculada. Adscrita a la Universidad de Granada.

${ }^{3}$ Departamento de Didactica de la Expresión Musical, Plastica y Corporal, Facultad de Ciencias de la Educacion, Universidad de Granada.

${ }^{4}$ Departamento de Metodos de Investigacion, Facultad de Humanidades y Educacion, Universidad de Granada (Campus de Melilla).

${ }^{5}$ Grupo de Investigacion GEDES. Departamento de Lenguajes y sistemas Informaticos. Universidad de Granada.

In Memoriam de Dr. Cipriano Romero Cerezo

\section{España}

Correspondencia: Felix Zurita Ortega. C/Sainz Cantero $n^{\circ} 1-2^{\circ} I z q d a$. CP/18002. Granada. España. E-mail: felixzo@ugr.es

(c) Education \& Psychology I+D+i and Editorial EOS (Spain) 


\section{Resumen}

Introducción. La implantación del Espacio Europeo de Educación Superior y la adopción del crédito ECTS, configuran un nuevo modelo metodológico para el profesorado y los estudiantes. Se resalta el protagonismo que debe asumir el estudiante en su aprendizaje, el apoyo y la orientación que debe recibir para la adquisición de competencias. Por tal motivo, el trabajo autónomo del estudiante y el uso de una plataforma de apoyo a la docencia son estrategias de interés para las innovaciones docentes.

Método. En este estudio hemos pretendido determinar, mediante un estudio descriptivo exploratorio, las percepciones que tienen nueve grupos de estudiantes $(n=290)$ sobre estas estrategias.

Resultados. Las valoraciones que efectúan sobre el trabajo autónomo y la utilización de la plataforma de apoyo a la docencia son positivas.

Discusión y Conclusiones. Ambas estrategias facilitan el desarrollo personal en la construcción de aprendizajes, constituyendo un medio de apoyo y de comunicación interpersonal bastante interesante para la implementación de los créditos ECTS.

Palabras Clave: Estrategias, Trabajo del estudiante, Plataforma de apoyo a la docencia, Innovación, Universidad. 


\title{
The student's work and use of a teaching support platform as methodological options at university
}

\begin{abstract}
Introduction. The implementation of the European Higher Education Area and the adoption of ECTS credit system establish a new methodological model for both teachers and students. It highlights the role that students must assume in their learning and the support and guidance they should be given in skills acquisition. For this reason, the student's independent study and the use of a support platform are valuable strategies for teaching innovations.
\end{abstract}

Method. In this study we sought to determine, through an exploratory, descriptive study, the perceptions of nine groups of students $(n=290)$ about these strategies.

Results. Their assessments on independent study and the use of the support platform for teaching are positive.

Discussion and Conclusion. Considering that they facilitate personal development in the construction of learning, being a support recourse, for interpersonal communication and useful for the implementation of ECTS.

Keywords: Strategies, student's work, the support platform for teaching, innovation, higher education. 


\section{Introducción}

Los principios de adaptación de la enseñanza al Espacio Europeo de Educación Superior (EEES) implican una reforma en la práctica docente y la articulación de estrategias metodológicas idóneas a este fin. La nueva tendencia cambia sustancialmente por la incidencia que tienen en las personas implicadas (profesor y estudiantes) y en los procesos a desarrollar (enseñanza y aprendizaje), es un modelo educativo basado en las interacciones didácticas del aprendizaje y en la persona que aprende (Álvarez, González y García, 2007; Arredondo y Norzagaray, 2009 y Prados, Cubero y De la Mata, 2010). La integración en el EEES supone que en la enseñanza universitaria debe desarrollarse en las mejores condiciones posibles, formando a estudiantes que deberán asumir en el futuro inmediato responsabilidades profesionales y sociales cada vez más complejas. La universidad debe de dar respuestas eficaces a las demandas sociales de calidad, percibiéndose una necesidad de cambio en el modelo que pueda realmente promover trasformaciones significativas en las metodologías docentes y ayude a la mejora de la práctica profesional (Correa y Paredes, 2009).

Para tal fin es fundamental una innovación docente que desplace su punto de gravedad de la enseñanza en priorizar el aprendizaje de competencias profesionales (González, Gilar y Ordoñez, 2008). El Proyecto Tuning Educational Structures in Europe (González y Wagenarr, 2003) en los nuevos modelos de diseños curriculares, sitúa a las competencias en el centro de la delimitación de los denominados "perfiles profesionales", así como el llamado nuevo paradigma educativo de "educación centrada en el aprendizaje del estudiante" (Bolivar, 2007). Esto implica un nuevo estilo de trabajo del docente, donde el desarrollo de las competencias supone dar respuestas a las necesidades actuales de los estudiantes para desenvolverse en sus aprendizajes, en su desarrollo personal y en el de orientación profesional (Yániz, 2008).

El trabajo académico deberá estar centrado en el aprendizaje del estudiante mediante la implicación activa en los planteamientos teóricos y en el desarrollo práctico de los procesos que lleva a cabo en su programa formativo (De Miguel, 2006). Igualmente, la Red Europea para garantizar la Calidad en la Educación Superior (ENQA, 2005) alude que la clave de la calidad depende de una mejora técnica de la planificación de la docencia, situando como eje el aprendizaje de los estudiantes. Nos encontramos ante la adopción de una nueva metodología docente que afecta al profesorado en su forma de enseñar y al alumnado en la manera de 
aprender, donde el aprendizaje es más personalizado y centrado en la visión constructivista y significativa para el estudiante (Rodríguez y Herrera, 2009). Una enseñanza centrada en los estudiantes supone que el profesor deberá prever el plan que deberán seguir, determinando los objetivos y organizando las actividades educativas para el aprendizaje en el logro de competencias de los estudiantes (Bolivar, 2007). En este contexto, Álvarez (2005) argumenta que el docente deja de ser fuente del conocimiento para desarrollar funciones de guía, orientador, asesor y facilitador de recursos y herramientas de aprendizaje en el trabajo más activo del estudiante para que adquiera competencias.

Para llevar a cabo estos procesos deberán adoptarse opciones metodológicas que propicien actividades de aprendizaje que se secuencian en el tiempo y que generan recursos necesarios para la adquisición de competencias. López-Aguado (2010) estima que el análisis de las estrategias que se adopten, cobra una gran importancia en función de los resultados esperados del aprendizaje en forma de competencias. Para tal fin las estrategias planificadas por el docente deben ser procedimientos a través de los cuales los estudiantes alcancen los objetivos en situaciones de aprendizaje concretos, con planteamientos flexibles, abiertos a las diferentes posibilidades, para que pueda personalizarse los ritmos y estilos de aprendizaje.

Con objeto de desarrollar una experiencia de innovación docente en la que se adopten opciones metodológicas centradas en los estudiantes y que contribuyan al desarrollo competencial, en este estudio (es parte de uno más amplio) nos hemos centrando en determinar los posicionamientos que tienen los estudiantes sobre el trabajo académico que desarrollan y el uso que hacen de una plataforma de apoyo a la docencia como estrategias de interés para el desarrollo del aprendizaje autónomo.

\section{El trabajo del estudiante}

El trabajo del estudiante conforman las actividades que realiza para preparar una asignatura, cumplir con los objetivos planteados y conseguir los resultados de aprendizaje (Rué, 2007). Incluye horas correspondientes a las clases lectivas (teóricas y prácticas), seminarios, tutorías, trabajos individuales y grupales, consulta de información y búsqueda documental en bibliotecas físicas o virtuales, preparación y realización de exámenes y las pruebas de evaluación (Fernández, 2006 y Romero, 2008). 
La adopción de los "créditos europeos" o ECTS (European Credits Transfer System) supone la cantidad de trabajo que el estudiante debe desarrollar en sus actividades presenciales y no presenciales de clase para lograr sus competencias. Representa, una unidad de medida del trabajo académico, la cantidad de trabajo del estudiante que debe desarrollar para cumplir con los objetivos del plan de estudios (Bolivar, 2007).

Sobre las clases teóricas, el informe de la Comisión para la Renovación de las Metodologías Educativas en la Universidad (Consejo de Coordinación Universitaria, 2006) indica que se suele emplear la lección magistral, aunque cada vez se acompaña más de la realización de ejercicios, resolución de problemas y discusión de casos prácticos, trabajos en grupos o debates. Se destaca las aportaciones de Rué (2007) y Romero, Zurita y Zurita (2010) sobre la búsqueda de una mayor participación activa de los estudiantes en la clase, en las que se debe propiciar que analicen, sinteticen y reflexionen sobre los contenidos en clase (estudio de casos, resolución de problemas,...) y la complementación con otras opciones metodológicas como las actividades grupales y la búsqueda documental tanto en las bibliotecas como a través de Internet.

Las clases prácticas son importantes para aplicar los conocimientos a situaciones concretas y son de gran interés para adquirir destrezas que favorecen el conocimiento de los métodos propios de cada titulación, propician la adquisición de competencias, desarrollan las habilidades técnicas, incentivan las habilidades de comunicación y proporcionan una visión global de la práctica profesional (De Miguel, 2006). La sociedad de conocimiento demanda una alta capacitación de los estudiantes para "aprender a aprender" y, en consecuencia, un modelo de enseñanza más centrado en el mismo proceso de aprendizaje (Chocarro, GonzálezTorres y Sobrino, 2007), combinándose con una implicación sobre los contenidos teóricos y prácticos de cada una de las materias que forman su proceso de enseñanza. Romero, Cepero, López y Ortega (2009) se posicionan sobre la implicación activa en las sesiones teóricas (participación en debates, foros y seminarios) y en el desarrollo práctico de la materia (participación en debates, análisis y crítica sobre la práctica simulada de una supuesta situación real).

El estudiante ha de ser un agente activo y protagonista de su aprendizaje, lo que conlleva nuevas metodologías docentes donde prime que no sólo deberá adquirir el conocimiento sino que su principal labor debe ser la de construirlo no sólo en las clases presenciales, además deberá dedicar un tiempo no presencial mediante el trabajo autónomo. En el trabajo 
del estudiante se prima el aprendizaje autónomo y guiado, desarrollando la capacidad de autorregulación del proceso, siguiendo su propio ritmo y acomodando el aprendizaje a sus particulares circunstancias e intereses, estableciendo la construcción de sus conocimientos de manera activa (Carpio, 2008). Esto conlleva la implicación y el compromiso del estudiante en su aprendizaje (Rosário, Mourão, Núñez, González-Pienda, Solano y Valle, 2007) y del profesor ante la nueva función docente y la acción tutorial (García, 2008). Así, el estudiante debe recibir el apoyo, ayuda y supervisión del profesor, el cual establece estrategias según las características personales de cada uno para que desarrolle su nivel de responsabilidad en su formación y en su evaluación (Romero, Zurita y Zurita, 2010).

\section{La plataforma de apoyo docente}

La utilización de nuevas Tecnologías de la Información y la Comunicación (TIC), como las plataformas de apoyo a la docencia, se contemplan como una necesidad de cara a la adaptación al EEES (García y Daneri, 2008), pueden convertirse en herramientas que ayudan a los estudiantes en su búsqueda personal, permitiéndole el acceso a la información, a utilizar los recursos disponibles y poder avanzar de manera autónoma en sus aprendizajes y en el logro de las competencias. La plataforma de apoyo a la docencia es una de las nuevas estrategias metodológicas que han permitido una transformación e impulso en la nueva cultura docente, así el empleo de portales Web de apoyo al aprendizaje, a la docencia y a la gestión de datos de los alumnos es interesante porque facilita al profesor la realización de algunas tareas docentes y de gestión repercutiendo en una mayor autonomía y autoaprendizaje del estudiante (Cañas, Calandria, Ortigosa, Ros y Díaz, 2007). Palacios (2005) va más allá cuando expresa que este elemento permite abordar contenidos que de otra forma serían muy áridos en su comprensión.

Dentro del contexto de la interacción didáctica, la integración de recursos tecnológicos en las metodologías activas que se promulgan desde el EEES posibilita la comunicación, colaboración y la disposición de conocimiento, constituyéndose en un recurso de aprendizaje constructivista (Benito y Cruz, 2005). La plataforma es una aplicación de ayuda a la enseñanza presencial, favorecedora de la comunicación fluida entre el docente y los estudiantes que introduce nuevas formas de enseñanza (Salinas, 2004) y donde se tiene acceso a la información y documentación necesaria permitiendo a ambos realizar un seguimiento de la evolución académica del alumno (Correa y Paredes, 2009). Su flexibilidad de horario y acceso continuo, 
la disponibilidad de la guía docente permite aclarar el camino a seguir por parte del estudiante en la adquisición de su aprendizaje, fomenta la comunicación entre profesor y estudiantes, aclarando las dudas de estos últimos, posibilitando la realización de tutorías virtuales en cualquier momento (Correa y De Pablos, 2009). También, de acuerdo a Álvarez et al. (2007), el uso de las nuevas tecnologías (plataforma, webs,...) incide en el desarrollo motivacional, por la posibilidad de la implicación y continuidad en los aprendizajes y en el logro de competencias.

\section{Contextualización y definición del problema}

La implantación de los nuevos títulos de grado en la Facultad de Ciencias de la Educación (Universidad de Granada) y el desarrollo actual de la experiencia piloto de los créditos ECTS en las titulaciones a extinguir del plan anterior, ha llevado a un grupo de profesores a buscar experiencias de innovación docente que muestren estrategias metodologías docentes acordes con las que se plantean desde el EEES. Para poder abordar la experiencia en unas condiciones lo más idóneas posibles, se ha pretendido conocer qué es lo que opinaban los protagonistas (los estudiantes) sobre las opciones metodológicas a emplear en los procesos de enseñanza y aprendizaje. Esta cuestión se ha considerado de gran interés, al entender que de esta manera, permitiría al profesorado gestionar los procesos de enseñanza y aprendizaje ajustados a un contexto determinado, mediante unos contenidos y un grupo de clase con unas características particulares (Zabalza, 2003), posibilitando un acercamiento a una enseñanza más efectiva al poder organizarla con más claridad al partir de los intereses y el compromiso de los estudiantes, propiciando un mejor clima y ambiente de aprendizaje (Bolivar, 2007).

Dentro del compromiso del profesorado de involucrarse en la experiencias de innovación docente y en la búsqueda de unas mejores condiciones de la enseñanza, se ha partido de los planteamientos teóricos que residen en que el eje fundamental de la docencia universitaria es el aprendizaje y su contribución a la adquisición de competencias, buscado conocer aquellos procedimientos que entienden los estudiantes que le ayudan para el aprendizaje autónomo y en el uso de herramientas (plataformas digitales) que faciliten el desarrollo de competencias (Salicetti y Romero, 2010). Por ello, nos planteamos un objetivo general a partir de la siguiente cuestión: ¿Podríamos establecer los posicionamientos que tienen los estudiantes de Magisterio, Educación Social y de Fisioterapia sobre su trabajo académico y el empleo de la plataforma de apoyo a la docencia en el desarrollo de sus aprendizajes? 


\section{Objetivos Especificos}

Atendiendo a lo planteado en la cuestion anterior, los objetivos especificos de este estudio se concretan en:

- Establecer las percepciones que tienen los estudiantes de magisterio (de cinco especialidades), educación social y los de fisioterapia sobre el trabajo que desarrollan y el uso de la plataforma de apoyo a la docencia.

- Analizar los posibles contrastes existentes entre el género y los diversos grupos de titulaciones sobre las apreciaciones de dichas metodologías.

\section{Método}

\section{Participantes}

La muestra es la composición natural de los grupos, que responden al 100\% de los estudiantes que se encontraban en clase en el momento de aplicar el cuestionario. Son un total de 290 sujetos de primer curso de las diplomaturas de Magisterio (distintas titulaciones) y Educación Social de la Facultad de Ciencias de la Educación de Granada, Magisterio en Educación Física de Melilla, además de estudiantes de Fisioterapia de Almería (España). Ver Figura 1 .

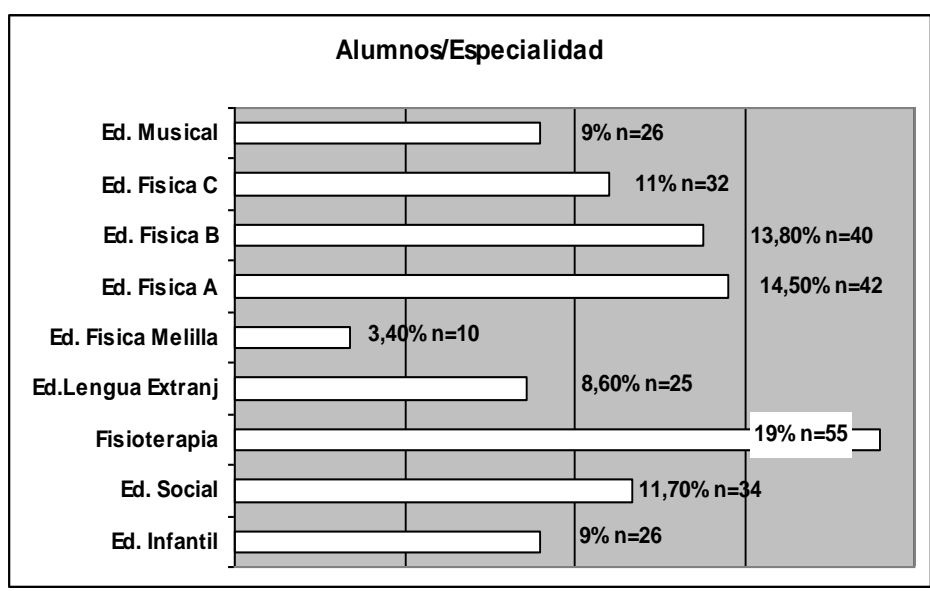

Figura 1. Distribución de la población de estudio por especialidad

Atendiendo al género, hay una proporción de $44.5 \%(\mathrm{n}=129)$ de hombres y un $55.5 \%$ $(n=161)$ de mujeres. Sobre la edad, la muestra fue muy heterogénea, considerando que eran 
estudiantes de primer curso, presentando un rango de edad entre los 17-19 años (47.9\%) y el resto muy distribuido conforme se aumenta la edad cronológica, hasta llegar a los 20 sujetos (6.9\%) de individuos mayores de 26 años.

\section{Diseño}

Este trabajo corresponde con un diseño no experimental de carácter descriptivo, exploratorio y transversal para determinar las percepciones que los estudiantes tienen sobre su trabajo en las actividades académicas y en el uso de una plataforma de apoyo a la docencia como estrategias metodológicas.

\section{Instrumentos}

Para obtener la información se ha recurrido a la técnica de encuesta del muestreo por conglomerados (grupos de individuos), empleando un cuestionario de respuestas cerradas según la escala Likert, de 15 ítems que valoran distintos aspectos sobre el trabajo personal y uso de la plataforma de docencia por parte del estudiante. Con el objeto de conocer, entender las variables a estudiar, obtener puntuaciones de cada uno de los ítems y poder comparar las valoraciones de diferentes estudiantes, nos hemos asegurado que el instrumento sea válido y fiable. Para la validez, nos hemos apoyado en una revisión teórica y documental para cubrir adecuadamente los distintos aspectos que pueden ser de interés para el trabajo autónomo y el uso de plataformas de apoyo a la docencia en el ámbito de la enseñanza universitaria, estableciendo diferentes ítems que, de esta manera, nos asegurábamos las relaciones teóricas relevantes con la validez del contenido que se pretendía medir. Además, mediante la técnica Delphy, se les pidió a ocho expertos que analizaran las preguntas, valorase su redacción, adecuación y relevancia del contenido. Por ello, las diversas cuestiones objeto de estudio se construyeron agrupándose en dos variables o dimensiones:

I. El trabajo del estudiante y su implicación activa con su aprendizaje (10 ítems).

II. Plataforma de apoyo a la docencia (5 ítems).

Los aspectos más relevantes de cada ítem del cuestionario construido lo contemplamos en el Anexo I. Teniendo en cuenta que se pretenden valorar los posicionamientos de varios grupos de estudiantes, nos valemos del factor intensidad, esgrimiendo una sucesión de respuesta tipo Likert con cuatro opciones sobre una serie de afirmaciones que cada estudiante 
deberá valorar tendiendo a una dirección positiva-favorable o negativa-desfavorable. En nuestro caso la codificación sería: (1) Nada, (2) Poco, (3) Bastante, (4) Mucho, donde una valoración positiva-favorable viene determinada por una mayor puntuación.

De acuerdo a la escala establecida, se calculó la correlación del ítem-total con todos los demás, obteniendo valores por encima de .30, oscilando entre el .31 del ítem I.9 a .51 del ítem II.2, que, según Morales, Urosa y Blanco (2003), tienen una buena discriminación. En cuanto a la fiabilidad de las preguntas se determinó con el coeficiente alfa de Cronbach (Bisquerra, 1987). En nuestro caso, el estadístico de fiabilidad para los 15 ítems del cuestionario es de .82, mientras que para cada una de las dimensiones es de .75 para los diez ítems del trabajo autónomo y .80 para los cinco ítems de la plataforma de apoyo a la docencia.

Para confirmar que las dimensiones teóricas establecidas en nuestro estudio coinciden con las dimensiones empíricas, se ha realizado un análisis factorial confirmatorio, obteniendo el siguiente resultado donde se puede comprobar que dimensiones teóricas y dimensiones empíricas coinciden. Para ello se ha utilizado el método de extracción de análisis de componentes principales y una rotación Varimax. Ver Tabla 1.

Tabla 1 .Analisis factorial de lasdimensiones teoricas

\begin{tabular}{|lcc|}
\hline \multicolumn{3}{|c|}{ Matriz de componentes rotados } \\
Items & \multicolumn{2}{c|}{ Componente } \\
& 1 & 2 \\
I.1 & & .514 \\
I.2 & & .676 \\
I.3 & & .646 \\
I.4 & & .694 \\
I.5 & & .407 \\
I.6 & & .503 \\
I.7 & & .518 \\
I.9 & & \\
I.10 & & .315 \\
I.11 & & .437 \\
II.1 & .757 & \\
II.2 & .796 & \\
II.3 & .769 & \\
II.4 & .674 & \\
II.5 & .614 & \\
\hline
\end{tabular}




\section{Procedimiento}

Los estudiantes en sus aulas y en sus horarios habituales de clase cumplimentaron el cuestionario que se le entregó por parte de un miembro del grupo investigador. Se contó con la autorización y la presencia en el aula del profesor responsable del grupo-curso. La participación de los estudiantes fue voluntaria y se les garantizó la confidencialidad de sus datos de identificación y demográficos. El cuestionario se contestó en 15 minutos.

\section{Análisis de los datos}

Para el análisis estadístico se empleó el paquete estadístico SPSS 15.0 y los resultados se obtuvieron mediante los estadísticos descriptivos básicos y la comparación de las medias de cada ítem. Para establecer las diferencias entre los diferentes grupos estudiados, previamente se ha efectuado la prueba de la normalidad de Kolmogorov-Smirnov y la de la igualdad de las varianzas de Levene, indicándonos, al verificarse la distribución normal de los ítems y no existir diferencias entre las varianzas, que es procedente efectuar las pruebas paramétricas, empleando la t para muestras independientes (T-Student) para conocer las diferencias entre ambos colectivos (hombres y mujeres). En el caso de los diversos grupos de titulaciones se han empleado la Anova de un factor aplicado la prueba de rango post hoc y las comparaciones múltiples por parejas, para determinar en qué sectores las medias difieren.

\section{Resultados}

Para la presentación de los resultados concernientes a las dos estrategias metodológicas, en primer lugar se focaliza en las medias y desviación típicas de los ítems del trabajo del estudiante y de la plataforma de apoyo a la docencia, a continuación se ha establecido el contraste entre ambas estrategias en relación al género y titulación.

\section{El trabajo del estudiante}

En el trabajo del estudiante (Tabla 2) destaca como más valorada la cuestión I.3, "Asistir a las clases prácticas y participar activamente en ellas (implicación, análisis, debate y crítica $)$ ” $(\mathrm{M}=3.43, \mathrm{DT}=.64)$. Seguida de lo expresado en los ítems I.1, “Asistir a las clases 
teóricas y escuchar atentamente las explicaciones que da el profesor en su lección magistral" $(\mathrm{M}=3.30, \mathrm{DT}=.68) ; \mathrm{I} .9$, "Emplear por parte del profesor diversas opciones metodológicas y de actividades en la clases" (M=3.29, DT= .71) y el I.7, "Considerar que la evaluación de la asignatura debe responder a criterios sobre el trabajo individual y la implicación activa que pueda tener el estudiante" $(\mathrm{M}=3.27, \mathrm{DT}=.65)$. El resto de los ítems oscilan entre el ítem I.2 $(\mathrm{M}=2.88, \mathrm{DT}=.079)$ y el ítem I.6 $(\mathrm{M}=3.22, \mathrm{DT}=.69)$, lo que muestra que no existen grandes oscilaciones entre las cuestiones relativas a esta opción metodológica.

Tabla 2 .Valores medios sobre el trabajo del estudiante y plataforma de apoyo a la docencia

\begin{tabular}{|c|c|c|c|c|c|c|c|c|c|c|c|c|c|c|c|}
\hline \multicolumn{11}{|c|}{ Trabajo del estudiante } & \multicolumn{5}{|c|}{$\begin{array}{l}\text { Plataforma de apoyo a la docen- } \\
\text { cia }\end{array}$} \\
\hline Ítem & I.1 & I. 2 & I. 3 & I. 4 & I.5 & I.6 & I.7 & I.8 & I.9 & I.10 & II.1 & II. 2 & II. 3 & II.4 & II.5 \\
\hline$M$ & 3.30 & 2.88 & 3.43 & 3.09 & 3.05 & 3.22 & 3.27 & 2.94 & 3.29 & 3.05 & 2.97 & 3.08 & 3.09 & 3.11 & 3.23 \\
\hline$D T$ & $(.68)$ & $(.79)$ & $(.64)$ & $(.72)$ & $(.71)$ & $(.69)$ & $(.65)$ & $(.88)$ & $(.71)$ & $(.81)$ & $(.66)$ & $(.71)$ & $(.71)$ & $(.76)$ & (.69) \\
\hline
\end{tabular}

Plataforma de apoyo a la docencia

En las puntuaciones otorgadas por los estudiantes a los diversos aspectos de la plataforma de apoyo a la docencia existen pocas oscilaciones entre la más puntuada la del ítem II.5, "Facilitar la comunicación con otros compañeros y con el profesor de la asignatura" $(\mathrm{M}=3.23, \mathrm{DT}=.69)$ y la que menos del II.1, “Apoyar y orientar al estudiante en el desarrollo del aprendizaje con respecto a la asignatura" $(\mathrm{M}=2.97, \mathrm{DT}=.66)$. En el resto de las cuestiones de los ítems II.4, II.3 y II.1 se mantienen con puntuaciones altas que difieren muy poco entre ellas.

Diferencias con respecto al género

En la comparativa por género en la distribución de las dos estrategias metodológicas, nos centramos primero en el trabajo del estudiante, que tal y como podemos apreciar en la Tabla 4, se han obtenido tan sólo diferencias significativas en el ítem I.9. "Emplear por parte del profesor diversas opciones metodológicas y de actividades en la clases" (t (288) = -1.99, $\mathrm{p}<.04)$, donde las estudiantes valoran mucho más esta opción que los estudiantes. En el resto 
de los nueve ítems no se han encontrado diferencias significativas respecto a la población femenina de la masculina.

Tabla 3. Comparativa del trabajo del estudiante y plataforma con respecto al género

\begin{tabular}{ccccccc}
\hline & \multicolumn{2}{l}{ Hombre (n= 129) } & \multicolumn{3}{c}{ Mujer (n= 161) } & $\begin{array}{r}\text { Prueba “t” en muestras } \\
\text { independientes }\end{array}$ \\
\cline { 2 - 7 } Ítem & $M$ & $D T$ & $M$ & $D T$ & $\mathrm{t}$ & Sig. \\
\hline I.1 & 3.29 & $(.71)$ & 3.30 & $(.65)$ & -.22 & .83 \\
I.2 & 2.82 & $(.78)$ & 2.93 & $(.79)$ & -1.12 & .26 \\
I.3 & 3.46 & $(.62)$ & 3.42 & $(.66)$ & .54 & .59 \\
I.4 & 3.11 & $(.66)$ & 3.07 & $(.76)$ & .47 & .64 \\
I.5 & 2.98 & $(.69)$ & 3.11 & $(.72)$ & -1.54 & .12 \\
I.6 & 3.19 & $(.67)$ & 3.25 & $(.72)$ & -.83 & .41 \\
I.7 & 3.19 & $(.65)$ & 3.32 & $(.65)$ & -1.68 & .09 \\
I.8 & 3.00 & $(.87)$ & 2.90 & $(.88)$ & .96 & .34 \\
I.9 & 3.20 & $(.73)$ & 3.37 & $(.68)$ & -1.99 & .04 \\
I.10 & 3.08 & $(.77)$ & 3.03 & $(.84)$ & .48 & .63 \\
II.1 & 2,88 & $(.62)$ & 3,04 & $(.69)$ & -2.04 & .04 \\
II.2 & 2,99 & $(.73)$ & 3,16 & $(.68)$ & -1.95 & .05 \\
II.3 & 2,98 & $(.73)$ & 3,17 & $(.68)$ & $-2,36$ & .01 \\
II.4 & 3,02 & $(.77)$ & 3,19 & $(.74)$ & -1.98 & .04 \\
II.5 & 3,16 & $(.72)$ & 3,28 & $(.65)$ & -1.44 & .15 \\
\hline
\end{tabular}

Sobre la plataforma de apoyo a la docencia, en la Tabla 2 se pueden comprobar cómo las valoraciones medias del género femenino son superiores al del género masculino en todos los ítems y esas diferencias son significativas en el ítem II.1, "Apoyar y orientar al estudiante en el desarrollo del aprendizaje con respecto a la asignatura", t (288) = -2.04, p <.04; II.3, “Tener acceso a recursos de interés práctico para la formación académica y para el futuro profesional", t $(288)=-2.36, \mathrm{p}<.01$ y II.4, "Acercar al conocimiento y dominio de las Nuevas Tecnologías de la Información y la Comunicación (NTIC), t (288) = -1.98, p <.04.

Diferencia entre grupos de titulaciones

Las puntuaciones de los ítems del trabajo del estudiante (Tabla 3) depararon diferencias entre los grupos, estas variaciones en la percepción de las distintas cuestiones se dieron en los ítems I.1,( Asistir a las clases teóricas y escuchar atentamente las explicaciones que da 
el profesor en su lección magistral) $\mathrm{F}(8,281)=2.55, \mathrm{p}<.01 ; \mathrm{I} .3$,(Asistir a las clases prácticas y participar activamente en ellas,implicación, análisis, debate y crítica sobre la práctica) $\mathrm{F}(8,281)=2.63, \mathrm{p}<.00$ y I.8,(Asumir un trabajo autónomo frente al exceso de contenidos teóricos y la primacía de clases magistrales que adoptan los profesores en sus clases) F (8, $281)=2.4, \mathrm{p}<.01 \mathrm{y}$ vinieron propiciadas por la diversidad en los valores medios.

Aplicando la prueba "post hoc" de comparaciones múltiples a posteriori de Bonferroni, se ha comprobado que esas diferencias se dan en el ítem I.1 entre Educación infantil y Educación Social $(\mathrm{p}<.01)$ y Educación Física C y Educación Social $(\mathrm{p}<.02)$. Así, se destaca $(\mathrm{M}=3.58, \mathrm{DT}=.50)$ del grupo de Educación Infantil y $(\mathrm{M}=3.50, \mathrm{DT}=.67)$ de Educación Física $\mathrm{C}$ respecto $(\mathrm{M}=2.94, \mathrm{DT}=.65)$ de Educación Social.

A pesar que en el ítem I.3, (Asistir a las clases prácticas y participar activamente en ellas,implicación, análisis, debate y crítica sobre la práctica) se dan diferencias significativas a nivel general, la oscilación de $(\mathrm{M}=3.16$, $\mathrm{DT}=.75)$ de Lengua Extranjera a $(\mathrm{M}=3.70, \mathrm{DT}=$ .48) de Educación Física Melilla, al contrastar por grupos se ha verificado que los grupos se encuentran asociados con respecto a este ítem. De manera similar se muestra el I.8, existe una oscilación de $(\mathrm{M}=3.50, \mathrm{DT}=.71)$ de Educación Física de Melilla frente $(\mathrm{M}=2.67, \mathrm{DT}=.86) \mathrm{de}$ Fisioterapia, no encontrando contrastes de disociación entre los grupos una vez que se le aplica la prueba de comparación múltiple.

En los valores medios de los ítems de la plataforma de apoyo a la docencia en relación con los grupos, no se observaron diferencias, por lo que la valoración es muy similar en todas las titulaciones. Ver Tabla 4.

Tabla 4. Distribución de valores medios de los ítems en los grupos y sus diferencias

\begin{tabular}{|c|c|c|c|c|c|c|c|c|c|c|c|c|c|c|c|c|}
\hline \multirow[t]{2}{*}{ Grupos } & \multicolumn{11}{|c|}{ Trabajo del estudiante } & \multicolumn{5}{|c|}{$\begin{array}{c}\text { Plataforma de apoyo a la docen- } \\
\text { cia }\end{array}$} \\
\hline & & I. 1 & I. 2 & I. 3 & I. 4 & I. 5 & I. 6 & I. 7 & I. 8 & I.9 & I. 10 & II. 1 & II. 2 & II. 3 & II. 4 & II. 5 \\
\hline Educación & $M$ & 3.58 & 2.85 & 3.27 & 3.08 & 3.00 & 3.15 & 3.27 & 2.73 & 3.23 & 3.23 & 3.04 & 3.12 & 3.38 & 3.31 & 3.35 \\
\hline Infantil & $D T$ & $(.50)$ & $(.54)$ & $(.72)$ & $(.79)$ & $(.80)$ & $(.78)$ & (.67) & (1.0) & $(.86)$ & $(.81)$ & $(.59)$ & $(.65)$ & (.64) & (.62) & $(.56)$ \\
\hline Educación & $M$ & 2.94 & 2.85 & 3.21 & 2.91 & 3.00 & 3.15 & 3.03 & 3.12 & 3.62 & 2.79 & 2.82 & 2.94 & 2.88 & 2.88 & 3.06 \\
\hline Social & $D T$ & (.65) & $(.78)$ & $(.59)$ & $(.75)$ & (.69) & $(.78)$ & (.67) & $(.88)$ & $(.65)$ & $(.84)$ & $(.83)$ & $(.85)$ & (.73) & $(.95)$ & (.65) \\
\hline ciotoroni & $M$ & 3.24 & 3.04 & 3.60 & 3.24 & 3.16 & 3.24 & 3.40 & 2.67 & 3.16 & 2.98 & 3.05 & 3.27 & 3.18 & 3.27 & 3.35 \\
\hline Fisioterapid & $D T$ & (.64) & (.77) & (.60) & (.69) & $(.60)$ & (.61) & $(.569$ & $(.86)$ & (.63) & $(.87)$ & $(.70)$ & (.65) & (.79) & (.65) & (.67) \\
\hline Lengua & $M$ & 3.20 & 2.40 & 3.16 & 2.76 & 3.24 & 3.16 & 3.24 & 2.84 & 3.08 & 3.08 & 3.20 & 3.24 & 3.24 & 3.20 & 3.12 \\
\hline
\end{tabular}




\begin{tabular}{ccccccccccccc|cccc} 
Extranjera & $D T$ & $(.64)$ & $(.82)$ & $(.75)$ & $(.78)$ & $(.72)$ & $(.80)$ & $(.60)$ & $(.85)$ & $(.70)$ & $(.70)$ & $(.50)$ & $(.59)$ & $(.59)$ & $(.64)$ & $(.67)$ \\
Educación & $M$ & 3.20 & 2.70 & 3.70 & 3.20 & 3.00 & 3.00 & 3.50 & 3.50 & 3.30 & 2.80 & 2.90 & 2.90 & 3.10 & 2.80 & 3.00 \\
Física & & & & & & & & & & & & & & & & \\
Melilla & $D T$ & $(.79)$ & $(1.06$ & $(.48)$ & $(.63)$ & $(.67)$ & $(.67)$ & $(.53)$ & $(.71)$ & $(.67)$ & $(.92)$ & $(.32)$ & $(.74)$ & $(.88)$ & $(.63)$ & $(.47)$ \\
Educación & $M$ & 3.40 & 2.93 & 3.48 & 3.17 & 2.88 & 3.43 & 3.26 & 2.98 & 3.31 & 3.19 & 2.86 & 3.02 & 3.00 & 2.98 & 3.29 \\
Física A & $D T$ & $(.70)$ & $(.84)$ & $(.67)$ & $(.69)$ & $(.83)$ & $(.67)$ & $(.70)$ & $(.84)$ & $(.68)$ & $(.67)$ & $(.52)$ & $(.68)$ & $(.62)$ & $(.89)$ & $(.77)$ \\
Educación & $M$ & 3.25 & 2.95 & 3.60 & 3.18 & 3.08 & 3.23 & 3.35 & 2.83 & 3.28 & 3.18 & 2.83 & 3.08 & 3.00 & 3.15 & 3.23 \\
Física B & $D T$ & $(.74)$ & $(.78)$ & $(.54)$ & $(.64)$ & $(.62)$ & $(.73)$ & $(.74)$ & $(.93)$ & $(.75)$ & $(.84)$ & $(.78)$ & $(.76)$ & $(.72)$ & $(.74)$ & $(.83)$ \\
Educación & $M$ & 3.50 & 2.78 & 3.50 & 2.97 & 2.91 & 3.13 & 3.13 & 3.22 & 3.31 & 3.03 & 2.97 & 2.81 & 2.91 & 3.06 & 3.19 \\
Física C & $D T$ & $(.67)$ & $(.71)$ & $(.57)$ & $(.65)$ & $(.78)$ & $(.61)$ & $(.66)$ & $(.79)$ & $(.53)$ & $(.78)$ & $(.59)$ & $(.74)$ & $(.64)$ & $(.80)$ & $(.69)$ \\
Educación & $M$ & 3.38 & 3.08 & 3.31 & 3.15 & 3.15 & 3.31 & 3.27 & 3.19 & 3.38 & 3.04 & 3.15 & 3.19 & 3.19 & 3.15 & 3.23 \\
Musical & $D T$ & $(.64)$ & $(.79)$ & $(.68)$ & $(.78)$ & $(.67)$ & $(.68)$ & $(.60)$ & $(.63)$ & $(.85)$ & $(.87)$ & $(.67)$ & $(.63)$ & $(.74)$ & $(.67)$ & $(.58)$ \\
Anova de & $\mathrm{F}$ & 2.55 & 1.88 & 2.63 & 1.53 & .98 & .82 & 1.30 & 2.40 & 1.52 & 1.02 & 1.4 & 1.62 & 1.69 & 1.38 & .81 \\
un factor & $\mathrm{Sig}$ & .01 & .06 & .00 & .14 & .45 & .59 & .24 & .01 & .15 & .41 & .20 & .12 & .09 & .20 & .59 \\
\hline
\end{tabular}

\section{Discusión y conclusiones}

\section{Percepciones de los estudiantes universitarios}

En el presente trabajo de investigación se ha verificado diversas apreciaciones y posicionamientos positivos sobre el trabajo del estudiante y sobre el uso de la plataforma de apoyo a la docencia.

\section{El trabajo del estudiante y su implicación activa}

El estudiante universitario considera de gran interés la asistencia a las clases prácticas y participar activamente en ellas. Este gran valor que le otorgan los estudiantes a las prácticas de las asignaturas también se puso de manifiesto por Andreu, Sanz y Serrat (2009) por la importancia del aprendizaje de los contenidos a través de la práctica. García y Álvarez (2007) y Rodríguez y Herrera (2009) obtuvieron que los estudiantes consideren que en este tipo de actividades les lleva a percibir la utilidad práctica de la asignatura y es de gran utilidad para el posterior futuro profesional. Struyven, Dochy, Janssens y Gielen (2008), comprobaron el mayor grado de satisfacción de los estudiantes cuando abordaron de manera activa y variada, determinadas situaciones basadas en la experiencia práctica. Confirmándose que los estudiantes prefieren una evaluación en la que se base en experiencias prácticas frente a otros modos más tradicionales de la enseñanza, donde primaba las aportaciones del docente frente al proceso enseñanza-aprendizaje del alumno, como ya han planteado De la Fuente y Justicia (2007). 
Otra cuestión de gran interés para los estudiantes es la asistencia a las clases teóricas y recibir la información pertinente por parte del profesor, considerándose que la enseñanza meramente expositiva resulta insuficiente para promover el cambio conceptual en los estudiantes, habría que proporcionarles unas orientaciones que promueva la capacidad de que se puedan implicar en sus aprendizajes (Romero, Zurita y Zurita, 2010). El docente debe de despertar el interés del estudiante y motivarlo, para que pueda desempeñar un papel activo en su proceso de aprendizaje tanto en las clases teóricas como en el desarrollo de casos prácticos (García y Álvarez, 2007); en este sentido Herrera, Jiménez y Castro (2011) indican que el alumnado motivado por los procesos de aprendizaje y que se siente autocompetente rinde mas en los diversos procesos. Las clases teóricas pueden ser de gran utilidad para despertar el interés y motivar a los estudiantes para sus aprendizajes, conforme a los hallazgos de Cabanach, Valle, Gerpe, Rodríguez, Piñeiro y Rosario (2009).

Los estudiantes consideran relevante que el profesor emplee diversas opciones metodológicas y actividades en la clase. Los estudios de Diez et al (2009) muestran como los estudiantes de educación esperan que el profesorado use distintas metodologías relacionadas con lecturas obligatorias, realización de trabajos, la utilización de recursos On-line, el uso de las nuevas tecnologías y una mayor participación de los estudiantes en el desarrollo de la asignatura. Struyven, Dochy, Janssens y Gielen (2008) dan a conocer la satisfacción de los estudiantes por el uso variado de métodos por ser más motivante. Gil, Bernaras, Elizalde y Arrieta (2009), en la comprobación que hicieron sobre distintas estrategias de aprendizaje y el tipo de motivación, valoran la utilidad de sus resultados para la adecuación metodológica de la enseñanza en el EEES. Por lo que se puede concretar que aspectos como la asistencia a clases teóricas, empleo de recursos metodológicos innovadores que mantengan altos grados de motivación e interés y una evaluación sobre la implicación personal del alumno son elementos valorados muy positivamente por los estudiantes analizados.

Para los estudiantes los procesos de evaluación deben responder a criterios del trabajo individual y a la implicación activa. Romero (2008) obtuvo resultados muy similares al considerar que la evaluación debe suponer una valoración continua del conocimiento, habilidades y actitudes de los estudiantes, reflejando la evolución del aprendizaje en cada momento del proceso. Sierens et al. (2009) encontraron correlaciones positivas entre aquellas metodologías docentes que propician el trabajo autónomo de los estudiantes que tienen apoyo necesario del 
profesor y las prestaciones que se esperan que logren los estudiantes, sobre todo cuando los procesos de evaluación se basan en las actividades realizadas por los estudiantes de manera autorregulada. Gallardo (2009) obtiene datos de interés sobre la importancia de la evaluación como un proceso continuo y sistemático, en donde se ofrece una retroinformación de los puntos fuertes y débiles, lo que permite adoptar medidas tanto por parte del estudiante como del profesor y buscando la motivación necesaria para lograr los resultados esperados.

\section{Plataforma de apoyo a la docencia}

A la plataforma de apoyo a la docencia los universitarios le otorgan un valor importante a todas las cuestiones planteadas en este estudio. Destaca que el uso de esta herramienta posibilita el intercambio comunicativo con sus compañeros y con el docente. De manera muy similar, Correa y Paredes (2009) y Salicetti y Romero (2010) encontraron los beneficios comunicativos entre los compañeros y el acceso a la información. Elgort, Smith y Toland (2008) confirman en los resultados obtenidos que en las plataformas educativas los estudiantes encuentran información útil, les permiten organizar conocimientos y compartir con el resto de los compañeros. Gallego, Gámiz y Romero (2009) aportan las percepciones que tienen los estudiantes sobre las herramientas telemáticas en la mejora del trabajo colaborativo y la facilitación del trabajo colectivo, permitiendo un ritmo propio de trabajo y la comunicación con el profesorado.

Los estudiantes de este estudio también resaltan que la plataforma debe servir para apoyar y orientar en el desarrollo de los aprendizajes. Resultados muy similares obtuvieron Coll, Mauri y Onrubia (2006) en donde los estudiantes destacan la utilidad del aula virtual para tener acceso de manera continuada a los recursos materiales, actividades y tareas de la asignatura, valorando la facilidad de acceso y las posibilidades de funcionamiento que ofrece. Las herramientas tecnológicas se consideran de gran interés por el apoyo que presta a la enseñanza presencial por los recursos didácticos, el apoyo tutorial y la facilitación de los aprendizajes, así Reinoso (2009) encontró en su estudio la importancia de la plataforma para promover la participación de los estudiantes y su implicación activa en sus propios aprendizajes al atribuirles un papel destacado en la construcción y estructuración de sus conocimientos. Por su parte, Palacios (2005) llega a establecer que es un elemento de enseñanza que le permite al alumnado un alto grado de independencia en su autoaprendizaje, puesto que es el mismo quien decide la sucesión de adquisición de conocimientos. Sogues, Gisbert e Isus (2007) detectan la importancia de las plataformas virtuales para el desarrollo de la tutoría, porque facilitan la comuni- 
cación entre tutor y estudiante sin necesidad de estar en el mismo espacio y tiempo, simplemente bastaría con aportar instrumentos de seguimiento y gestión.

\section{Diferencias existentes entre el género y las diversas titulaciones}

En el trabajo del estudiante se ha verificado que las alumnas son más partidarias que los alumnos que el profesor emplee diversas opciones metodológicas y actividades en la clases. Esta evidencia es similar a la obtenida por García (2005) al poner de manifiesto la motivación de las estudiantes por abordar más profundamente sus aprendizajes a través del uso de diferentes estrategias de aprendizaje. Cano (2000) encontró mayores actitudes de las alumnas hacia las actividades de aprendizaje que los alumnos, lo que confiere que las mujeres son más activas en la configuración de sus aprendizajes. Con respecto al género y el uso de la plataforma de apoyo a la docencia, las alumnas ofrecen posicionamientos más positivos que los alumnos para hacer uso de estas herramientas, consideran que la plataforma puede ser un gran apoyo para desarrollar aprendizajes de la asignatura, acceder a recursos de interés práctico para la formación académica y para el futuro profesional y les permite un conocimiento y dominio de las NTIC.

Analizado los posicionamientos de las nueve especialidades objeto de estudio y considerando aquellas cuestiones que son claramente significativas, apreciamos las diferencias entre tres ítems y los grupos en relación con el trabajo personal del alumnado. Destaca el interés de los estudiantes de Educación Infantil y Educación Física C por asistir a las clases teóricas presenciales y escuchar las explicaciones que les proporcionan el profesor de la asignatura, al contrario que los estudiantes de Educación Social que no valoran tanto la asistencia a clase o la influencia de los docentes, siendo más partidarios del trabajo autónomo.

En líneas generales consideran que se debe emplear por parte del profesor diversas opciones metodológicas y de actividades en las clases, que atienda a las necesidades personales de los estudiantes que tengan una mayor aplicación y utilidad práctica. Estos contrastes coinciden con De la Fuente y Sánchez (2000), en donde los estudiantes rechazan parcialmente los enfoques teóricos que se hacen desde la universidad, adoptando una actitud puramente instrumental y de realización de actividades más prácticas. Martínez (2007) encontró en el portafolio una oportunidad para que los estudiantes de Educación Social efectúen un aprendizaje más reflexivo, autónomo y participativo. Igualmente Herrera y Lorenzo (2009) hallaron, en 
las opiniones de los estudiantes, que gran parte de los profesores no emplean estrategias y metodologías docentes que fomenten el aprendizaje autónomo, colaborativo y crítico del alumnado, sino un aprendizaje mecánico y más cercano a lo memorístico. A tal efecto, proponen la implicación del profesorado en procesos de innovación docente que propicien situaciones de aprendizajes dinámicas, constructivas y significativas para los estudiantes.

En la plataforma de apoyo a la docencia no se han encontrado diferencias entre las diversas titulaciones, destacando la estrecha cohesión hallada entre los nueve grupos analizados y sus percepciones altas con respecto a las distintas ideas que se contemplan en los ítems.

\section{Referencias}

Álvarez, B., González, C., y García, N. (2007). La motivación y los métodos de evaluación como variables fundamentales para estimular el aprendizaje autónomo. Revista de Docencia Universitaria. Recuperado de http://www.um.es/ead/Red_U/2/alvarez.pdf (consultado el 3/02/09).

Álvarez, M. B. (2005). Adaptación del método docente al Espacio Europeo de Educación Superior: La motivación de los alumnos como instrumento clave. Estudios Sobre Educación, 9, 107-126.

Andreu, Ll., Sanz, M., y Serrat, E. (2009). Una propuesta de renovación metodológica en el marco del Espacio Europeo de Enseñanza Superior: los pequeños grupos de investigación cooperativos. Revista Interuniversitaria de Formación del Profesorado, 12 (3), 111-126. Recuperado de http//www.aufop.com (consultado el 14/03/10).

Arredondo, J.J y Nozargaray, C.C (2009). Evaluación del laboratorio de orientación educativa como escenario de práctica en un modelo curricular basado en competencias. Electronic Journal of Research in Educational Psychology, 7(3), 985-1006.

Benito, A. y Cruz, A. (2005). Nuevas claves para la docencia universitaria. Madrid: Nancea.

Bisquerra, R. (1987). Introducción a la estadística aplicada a la investigación educativa. Un enfoque informático con los paquetes BHDP y SPSSX. Barcelona: PPU.

Bolivar, A. (2007). La planificación por competencias en la reforma de Bolonia de la educación superior: un análisis crítico. ETD - Educação Temática Digital , Campinas, v.9, n. esp., 68-94. 
Cabanach, R. G., Valle, A., Gerpe, M. G., Rodríguez, S., Piñeiro, I. y Rosario, P. (2009). Diseño y validación de un cuestionario de gestión motivacional. Revista Psicodidáctica, 14(1), 29-47.

Cano, F. (2000). Diferencias de género en estrategias y estilos de aprendizaje. Psicothema, 12 (3), 360-367.

Cañas, A., Calandria, D. J., Ortigosa, E. M., Ros, E., y Díaz, A. F. (2007). SWAD: Web System for Education Support. . En J. M. S. P. B. Fernández-Manjón, J. A. GómezPulido, M. A. Vega Rodríguez, J. Bravo-Rodríguez (Eds.), Computers And Education: E-learning - from Theory to Practice (pp 133-142).Dordrecht: Springer.

Carpio, C. (2008). Métodos de enseñanza-aprendizaje aplicables en Magisterio en el marco del espacio europeo de Educación Superior. Docencia e Investigación. Revista de la Escuela Universitaria de Magisterio de Toledo, 33(18).

Coll, C., Mauri, T. y Onrubia, J. (2006). Análisis y resolución de casos-problema mediante el aprendizaje colaborativo. Revista de Universidad y Sociedad del Conocimiento, 3 (2), $29-41$.

Correa, J.M y Paredes, J. (2009). Cambio tecnológico, usos de plataformas de e-learning y transformación de la enseñanza en las universidades españolas: la perspectiva de los profesores. Revista Psicodidáctica, 14(2), 261-278.

Chocarro, E. González-Torres, M.C. y Sobrino, A. (2007). Nuevas orientaciones en la formación del profesorado para una enseñanza centrada en la promoción del aprendizaje autorregulado de los alumnos. Estudios Sobre Educación, 12, 81-98.

Consejo de Coordinación Universitaria (2006). Propuestas metodológicas para la renovación de las metodologías educativas. Madrid: Ministerio de Educación y Ciencia. 286 p. Recuperado de http://www.mec.es/educa/ccuniv/html/metodologias/ (consultado el 10/02/08).

Correa, J.M. y De Pablos, J. (2009). Nuevas tecnologías e innovación docente. Revista Psicodidáctica, 14(1), 133-145.

Correa, J.M. y Paredes, J. (2009). Cambio tecnológico, usos de plataformas de e-learning y transformación de la enseñanza en las universidades españolas: la perspectiva de los profesores. Revista Psicodidáctica, 14(2), 261-278.

Díez, M.C., Pacheco, D.I., García, J.N., Martínez, B., Robledo, P., Álvarez, M.L. et al. (2009). Percepción de los estudiantes universitarios de educación respecto al uso de metodologías docentes y el desarrollo de competencias ante la adaptación al EEES: datos de la Universidad de Valladolid. Aula Abierta, 37 (1), 45-56. 
De Miguel, M. (2006). Metodologías para organizar el aprendizaje. Segundo objetivo del Espacio Europeo de Educación Superior. Revista Interuniversitaria de Formación del Profesorado, 20 (3), 71-91.

De la Fuente, J. y Justicia, F. (2007). El modelo DINEPRO de regulación de la enseñanza y aprendizaje: Avances recientes. Electronic Journal of Research in Educational Psychology, 5, 535-564.

De la Fuente, G. y Sánchez, M. E. (2000). La socialización anticipada en la Universidad. El caso de los estudiantes de Magisterio y Educación Social. Revista de Educación, 321, 269-290.

Elgort, I., Smith, A. G., y Toland, J. (2008). Is Wiki an effective platform for group course work? AJET: Australasian. Journal of Educational Technology, 24(2), 195-210.

ENQA (European Association For Quality Assurance in Higher Education) (2005). Standars and guidelines for Quality Assurance in the European Higher Education Area. Helsinki: ENQA. Recuperado de http://www.bologna-bergen2005.no/Docs/ 00-Main doc/050221_ENQA_report.pdf (consultado el 14/03/2009).

Fernández, A. (2006). Metodologías activas para la formación de competencias. Educatio siglo XXI, 24, 35- 6.

Gallardo, A. (2009). Aproximación al estudio de evaluación en la universidad: un análisis exploratorio con alumnos semipresenciales. Contextos Educativos, 12, 77-93.

Gallego, M. J., Gámiz, V., Romero, M. A. (2009). Perfil de entrada de los estudiantes universitarios ante las plataformas de apoyo a la práctica basadas-en-Internet. REIFOP, 12 (3), 153-164. Recuperado de http//www.aufop.com (consultado el 14/03/2010).

García, A.B. (2005). Estudio de los enfoques de aprendizaje en estudiantes de Magisterio y Psicopedagogía. Electronic Journal of Research in Educational Psychology, 3 (6), 109-126.

García, N. (2008). La función tutorial de la Universidad en el actual contexto de la Educación Superior. Revista Interuniversitaria de Formación del Profesorado, 22 (1), 21-48.

García, A. y Daneri, M. (2008). La integración de las tecnologías de la información y comunicación en la enseñanza universitaria: cómo afrontan los profesores el cambio al Espacio Europeo de Educación Superior. En R. I. Roig y J.E. Blasco (Coord.). Investigación e innovación en el conocimiento educativo actual (pp. 129-150). Alcoy (Alicante): Marfil.

García, N y Álvarez, M. B (2007). La motivación del alumnado a través de la satisfacción con la asignatura. Efecto sobre el rendimiento. Estudios Sobre Educación, 13, 89-112. 
Gil, P., Bernaras, E., Elizalde, L. M. y Arrieta, M. (2009).Estrategias de aprendizaje y patrones de motivación del alumnado de cuatro titulaciones del Campus de Gipuzkoa. Infancia y Aprendizaje, 32 (3), 329-341.

González, J. y Wagenaar, R. (2003). Tuning Educational Structure in Europe. Informe final. Bilbao: Universidad de Deusto. Universidad de Groningen / ANECA.

González, C., Gilar, R. y Ordoñez, T. (2008). El Dossier de aprendizaje autónomo del alumnado como una herramienta de trabajo en el marco del Espacio Europeo de Educación Superior. En R. I. Roig y J.E. Blasco (Coord.). Investigación e innovación en el conocimiento educativo actual (pp. 151-166). Alcoy (Alicante): Marfil.

Herrera, L. y Lorenzo, O. (2009). Estrategias de aprendizaje en estudiantes universitarios. Un aporte a la construcción del Espacio Europeo de Educación Superior. Educación y Educadores, 12 (3), 75-98.

Herrera, L., Jiménez, G. y Castro, A. (2011). Aprendizaje del alumnado universitario de primer y ultimo curso de las titulaciones de Maestro y Psicologia. Electronic Journal of Research in Educational Psychology, 9(2), 659-692.

López-Aguado, M. (2010). Diseño y análisis del Cuestionario de Estrategias de Trabajo Autónomo (CETA) para estudiantes universitarios. Revista de Psicodidáctica, 15(1), 77-99.

Martínez, S. (2007). Una experiencia de innovación del portafolio del alumno, en la diplomatura de educación social, desde el marco de la educación superior en Europa. Educatio Siglo XXI, 25, 125-144.

Morales, P., Urosa, B. y Blanco, A. (2003). Construcción de escalas de actitudes tipo Likert. Madrid: La Muralla.

Palacios, S. (2005). Variables psicodidácticas de las nuevas tecnologías. Revista Psicodidáctica, 10(2), 81-96.

Prados, M.M., Cubero, M y De la Mata, M. (2010). ¿Mediante que estructuras interactivas se relacionan profesorado y alumnado en las aulas universitarias?. Electronic Journal of Research in Educational Psychology 8(1): 163-194.

Reinoso, A. J. (2009) Análisis de la incorporación de una plataforma Wiki a la docencia de la asignatura "Nuevas Tecnologías de la Información”. Red U - Revista de Docencia Universitaria. Número Monográfico V. Número especial dedicado a WIKI y educación superior en España (II parte). Recuperado de http://www.um.es/ead/Red_U/m5/ (consultado el 22/12/2010). 
Rodríguez, C. y Herrera, L. (2009). Análisis correlacionar-predictivo de la influencia de la asistencia a clase en el rendimiento académico universitario. Estudio de caso en una asignatura. Profesorado. Revista de currículum y formación del profesorado. 13 (2). Recuperado de http://www.ugr.es/ recfpro/rev132COL4.pdf (consultado el 16/11/09).

Romero, C. (2008). El portafolios y el trabajo de grupo: una experiencia del crédito ECTS en la formación del magisterio especialista en Educación Física. REIFOP, 9 (2). Recuperado de http://www.aufop.com/aufop/revistas/arta/digital/93/446 (consultado el 20/01/09).

Romero, C.; Cepero, M.; López, M.; Ortega, J.L. (2009). Evaluation of Methodological Teaching Strategies for Students of Different Specializations in Education Sciences, Social Education and Physiotherapy. The International Journal of Interdisciplinary Social Sciences, 4 (9), 139-154.

Romero, C., Zurita, F. y Zurita, F. (2010). Autonomía y orientación en el Espacio Europeo de Educación Superior mediante el portafolio y la tutoría. ESE, Estudios Sobre Educación, 19, 261-282.

Rosário, P., Mourâo, R., Núñez, J.C., González-Pienda, J., Solano, P. y Valle, A. (2007). Eficacia de un programa instruccional para la mejora de procesos y estrategias de aprendizaje en la enseñanza superior. Psicothema, 19(3), 422- 427.

Rué, J. (2007). Enseñar en la Universidad. El EEES como reto para la Educación Superior. Madrid: Narcea.

Salicetti, A. y Romero, C. (2010). La plataforma de apoyo a la docencia como opción metodológica para el aprendizaje de competencias. Educación, Revista de la Universidad de Costa Rica, 34(1), 83-100.

Salinas, J. (2004). Cambios metodológicos con las TIC. Estrategias didácticas y entornos virtuales de enseñanza-aprendizaje. Bordón, 56, 469-481.

Sierens, E., Vansteenkiste, M., Goossens, L., Soenens, B. y Dochy, F. (2009). The synergistic relationship of perceived autonomy support and structure in the prediction of selfregulated learning. British Journal of Educational Psychology, 79 (1), 57-68.

Sogues, M., Gisbert, M. e Isus, S. (2007). E-tutoría: uso de las tecnologías de la información y comunicación para la tutoría académica universitaria. Teoría de la Educación: Educación y Cultura en la Sociedad de la Información, 8 (2), 31-54.

Struyven, K., Dochy, F., Janssens, S. y Gielen, S. (2008). Students' experiences with contrasting learning environments: The added value of students' perceptions. Learning Environments Research, 11(2), 83, 109. 
Yániz, C. (2008). Las competencias en el currículo universitario: implicaciones para diseñar el aprendizaje y para la formación del profesorado. Red U. Revista de Docencia Universitaria. Recuperado de http: //www.redu.m.es/Red_U/m1, 1 (consultado el 08/12/10).

Zabalza, M. A. (2003). Competencias docentes del profesorado universitario: calidad del desarrollo profesional. Madrid: Narcea. 


\title{
Anexo I. Cuestionario de estrategias metodológicas valoradas por escala Likert
}

\author{
I. El trabajo del estudiante \\ I.1. Asistir a las clases teóricas y escuchar atentamente las explicaciones que da el profesor en su lección ma- \\ gistral. \\ I.2. Participar en los debates, foros, seminarios y talleres que se organicen en torno a la asignatura. \\ I.3. Asistir a las clases prácticas y participar activamente en ellas (implicación, análisis, debate y crítica sobre \\ la práctica).
}

I.4. Participar en el debate, análisis y crítica sobre la simulación de una supuesta situación real y sobre el modelo de intervención didáctica en la clase práctica.

I.5. Dedicar un tiempo al estudio, a la búsqueda documental y la obtención de información sobre los contenidos y otras actividades no presenciales de la asignatura.

I.6. Realizar actividades de clase que atiende a las necesidades personales del estudiante, tienen una utilidad práctica para una posterior inserción laboral y social

I.7. Considerar que la evaluación de la asignatura debe responder a criterios sobre el trabajo individual y la implicación activa que pueda tener el estudiante

I.8. Asumir un trabajo autónomo frente al exceso de contenidos teóricos y la primacía de clases magistrales que adoptan los profesores en sus clases

I.9. Emplear por parte del profesor diversas opciones metodológicas y de actividades en la clases

I.10. Preparar, exponer y debatir trabajos en clase conjuntamente con otros compañeros

II. La plataforma de apoyo a la docencia

II.1. Apoyar y orientar al estudiante en el desarrollo del aprendizaje con respecto a la asignatura.

II.2. Disponer y compartir información relevante de la asignatura.

II.3. Tener acceso a recursos de interés práctico para la formación académica y para el futuro profesional.

II.4. Acercar al conocimiento y dominio de las Nuevas Tecnologías de la Información y la Comunicación (NTIC)

II.5. Facilitar la comunicación con otros compañeros y con el profesor de la asignatura. 